Commun. Korean Math. Soc. 28 (2013), No. 4, pp. 735-740

http://dx.doi.org/10.4134/CKMS.2013.28.4.735

\title{
REAL QUADRATIC FUNCTION FIELDS OF MINIMAL TYPE
}

\author{
Dongho Byeon, Jiae Keem, and Sangyoon Lee
}

\begin{abstract}
In this paper, we will introduce the notion of the real quadratic function fields of minimal type, which is a function field analogue to Kawamoto and Tomita's notion of real quadratic fields of minimal type. As number field cases, we will show that there are exactly 6 real quadratic function fields of class number one that are not of minimal type.
\end{abstract}

\section{Introduction}

Let $q$ be an odd prime and $k=\mathbb{F}_{q}$ the finite field of order $q$. Let $D \in k[x]$ be a monic square-free polynomial of even degree and $K=k(x)(\sqrt{D})$ the real quadratic function field over $k$. Let $O_{K}=k[x]+k[x] \sqrt{D}$ be the integral closure of $k[x]$ in $K, h\left(O_{K}\right)$ the ideal class number of $O_{K}$, and $\epsilon_{K}=t_{D}+u_{D} \sqrt{D}$ the fundamental unit of $K$.

Let $D \in k[x]$ be a monic square-free polynomial of even degree. Then $\sqrt{D}$ has a continued fraction expansion

$$
\sqrt{D}=\left[a_{0}, \overline{a_{1}, a_{2}, \ldots, a_{l-1}, 2 a_{0}}\right],
$$

where the sequence of non-constant polynomials $a_{1}, \ldots, a_{l-1}$ is palindromic, that is, $a_{l-i}=a_{i}$ for $1 \leq i \leq l-1$. Here $l$ is the period of $D$.

For a given positive integer $l>1$ and a palindromic sequence of nonconstant polynomials $a_{1}, \ldots, a_{l-1}$ in $k[x]$, let $S_{k}\left(l ; a_{1}, \ldots, a_{l-1}\right)$ be the set of all monic square-free polynomials $D$ of even degree in $k[x]$ such that $\sqrt{D}=$ $\left[a_{0}, \overline{a_{1}, \ldots, a_{l-1}, 2 a_{0}}\right]$ for some non-constant polynomial $a_{0}$ in $k[x]$.

In this paper, we will show the following theorem, which is a function field analogue to [1] and [4].

Theorem 1.1. Let $q$ be an odd prime and $k=\mathbb{F}_{q}$. For a given positive integer $l>1$ and a palindromic sequence of non-constant polynomials $a_{1}, \ldots, a_{l-1}$ in

Received January 9, 2013.

2010 Mathematics Subject Classification. 11R58.

Key words and phrases. function field, class number.

This work was supported by KRF-2010-0026473 and partially supported by NRF2013R1A1A2007694. 
$k[x], \operatorname{deg} D>\operatorname{deg} u_{D}$ for all $D \in S_{k}\left(l ; a_{1}, \ldots, a_{l-1}\right)$ with one possible exception. If the exception exists, then it has the least degree in $S_{k}\left(l ; a_{1}, \ldots, a_{l-1}\right)$.

The following definition is a function field analogue to Kawamoto and Tomita's notion of real quadratic fields of minimal type in [5].

Definition 1.2. Let $q$ be an odd prime and $k=\mathbb{F}_{q}$. For a given positive integer $l>1$ and a palindromic sequence of non-constant polynomials $a_{1}, \ldots$, $a_{l-1}$ in $k[x]$, if $S_{k}\left(l ; a_{1}, \ldots, a_{l-1}\right)$ has the exception $D$ in Theorem 1.1 , then we call $K=k(x)(\sqrt{D})$ a real quadratic function field of minimal type over $k$.

In [5], Kawamoto and Tomita proved that there exist exactly 51 real quadratic fields of ideal class number one that are not of minimal type with one more possible exception. In this paper, we will show the following similar theorem.

Theorem 1.3. Let $q$ be an odd prime and $k=\mathbb{F}_{q}$. If $q=3$ or 5 , there are exactly 6 real quadratic function fields over $k$ with ideal class number one that are not of minimal type. If $q \geq 7$, all real quadratic function fields with ideal class number one is of minimal type.

\section{Proof of Theorem 1.1}

Let $q$ be an odd prime and $k=\mathbb{F}_{q}$. For a continued fraction expansion in $k\left(\left(\frac{1}{x}\right)\right)$

we define for $i \in \mathbb{N}_{0}$,

$$
\left[a_{0}, a_{1}, a_{2}, \ldots\right]
$$

$$
\begin{array}{ll}
p_{-2}:=0, & p_{-1}:=1, \quad p_{i}:=a_{i} p_{i-1}+p_{i-2}, \\
q_{-2}:=1, & q_{-1}:=0, \quad q_{i}:=a_{i} q_{i-1}+q_{i-2} .
\end{array}
$$

Then we have for $i \in \mathbb{N}_{0}$,

$$
\begin{aligned}
& \frac{p_{i}}{q_{i}}=\left[a_{0}, a_{1}, a_{2}, \ldots, a_{i}\right], \\
& p_{i} q_{i-1}-p_{i-1} q_{i}=(-1)^{i-1}, \\
& p_{i+1}=a_{i+1} p_{i}+p_{i-1}, \\
& q_{i+1}=a_{i+1} q_{i}+q_{i-1} .
\end{aligned}
$$

We call $\frac{p_{i}}{q_{i}}$ the $i$-th approximant of the continued fraction $\left[a_{0}, a_{1}, a_{2}, \ldots\right]$.

Let $a_{1}, \ldots, a_{l-1}$ be a palindromic sequence of non-constant polynomials in $k[x]$. Suppose that there exists a monic square-free polynomial $D$ of even degree and $a_{0} \in k[x]$ such that

$$
\sqrt{D}=\left[a_{0}, \overline{a_{1}, a_{2}, \ldots, a_{l-1}, 2 a_{0}}\right] .
$$

Then we have

$$
\sqrt{D}=a_{0}+\beta
$$


where $\beta=\left[0, \overline{a_{1}, a_{2}, \ldots, a_{l-1}, 2 a_{0}}\right]$. Let $\frac{p_{i}}{q_{i}}$ be the $i$-th approximant of the continued fraction $\left[0, \overline{a_{1}, a_{2}, \ldots, a_{l-1}, 2 a_{0}}\right]$. Then we have

$$
p_{l-1}=q_{l-2}
$$

and

$$
\sqrt{D}=\sqrt{a_{0}^{2}+\frac{2 a_{0} p_{l-1}+p_{l-2}}{q_{l-1}}}
$$

(cf. [7, p. 338]). We note that $\operatorname{deg} a_{0}^{2}>\operatorname{deg} \frac{2 a_{0} p_{l-1}+p_{l-2}}{q_{l-1}}$. If we let $v:=$ $\frac{2 a_{0} p_{l-1}+p_{l-2}}{q_{l-1}}$, then we have $v \in k[x]$ and

$$
2 a_{0} p_{l-1}+p_{l-2}=v q_{l-1} .
$$

So we have

$$
2 a_{0} p_{l-1}-v q_{l-1}=-p_{l-2}=(-1)^{l+1} p_{l-2}\left(p_{l-1} q_{l-2}-p_{l-2} q_{l-1}\right)
$$

and

$$
p_{l-1}\left(2 a_{0}+(-1)^{l} p_{l-2} q_{l-2}\right)=q_{l-1}\left(v+(-1)^{l} p_{l-2}^{2}\right) .
$$

Since $p_{l-1}$ and $q_{l-1}$ are relatively prime, there exists $w \in k[x]$ such that

$$
2 a_{0}+(-1)^{l} p_{l-2} q_{l-2}=w q_{l-1}
$$

and

$$
v+(-1)^{l} p_{l-2}^{2}=w p_{l-1}
$$

Thus we have

$$
D=\left(\frac{w q_{l-1}+(-1)^{l-1} p_{l-2} q_{l-2}}{2}\right)^{2}+w p_{l-1}+(-1)^{l-1} p_{l-2}^{2}
$$

and obtain the following lemma.

Lemma 2.1. Let $q$ be an odd prime, $k=\mathbb{F}_{q}$, and $D$ a monic square-free polynomial of even degree in $k[x]$. Let $l>1$ be a positive integer, $a_{1}, \ldots, a_{l-1}$ a palindromic sequence of non-constant polynomials in $k[x]$, and $\frac{p_{i}}{q_{i}}$ the $i$-th approximant of the continued fraction $\left[0, a_{1}, a_{2}, \ldots, a_{l-1}\right]$. Suppose that there exists $a_{0} \in k[x]$ such that

$$
\sqrt{D}=\left[a_{0}, \overline{a_{1}, a_{2}, \ldots, a_{l-1}, 2 a_{0}}\right] .
$$

Then there exists $w \in k[x]$ such that

$$
D=\left(\frac{w q_{l-1}+(-1)^{l-1} p_{l-2} q_{l-2}}{2}\right)^{2}+w p_{l-1}+(-1)^{l-1} p_{l-2}^{2} .
$$

The following lemma is well known. 
Lemma 2.2. Let $q$ be an odd prime and $k=\mathbb{F}_{q}$. Let $D$ be a monic square-free polynomial of even degree in $k[x]$ such that $\sqrt{D}=\left[a_{0}, \overline{a_{1}, a_{2}, \ldots, a_{l-1}, 2 a_{0}}\right]$ and $\frac{p_{i}}{q_{i}}$ the $i$-th approximant of the continued fraction. Let $K=k(x)(\sqrt{D})$ be the real quadratic function field over $k$ and $\epsilon_{K}$ the fundamental unit of $K$. Then

$\epsilon_{K}= \begin{cases}p_{l-1}+q_{l-1} \sqrt{D} & \text { if } l \text { is eqaul to the quasi-period of } D, \\ p_{\frac{l}{2}-1}+q_{\frac{l}{2}-1} \sqrt{D} & \text { if } l \text { is eqaul to two times of the quasi-period of } D .\end{cases}$

Remark. It is well known that the period of $D$ is equal to the quasi-period or two times of the quasi-period of $D$. For detail and definition of quasi-period, see $[8]$.

Proof of Theorem 1.1. Let $q$ be an odd prime and $k=\mathbb{F}_{q}$. For a given positive integer $l>1$ and a palindromic sequence of polynomials $a_{1}, \ldots, a_{l-1}$ in $k[x]$, suppose that $D \in S_{k}\left(l ; a_{1}, \ldots, a_{l-1}\right)$. Then by Lemma 2.1 , we have

$$
D=\left(\frac{w q_{l-1}+(-1)^{l-1} p_{l-2} q_{l-2}}{2}\right)^{2}+w p_{l-1}+(-1)^{l+1} p_{l-2}^{2}
$$

for some $w \in k[x]$, where $\frac{p_{i}}{q_{i}}$ the $i$-th approximant of the continued fraction $\left[0, a_{1}, a_{2}, \ldots, a_{l-1}\right]$. So if $\operatorname{deg} w q_{l-1} \neq \operatorname{deg} p_{l-2} q_{l-2}$, then we have

$$
\begin{aligned}
\operatorname{deg} D & \geq \operatorname{deg} a_{0}^{2} \\
& =\operatorname{deg}\left(\frac{w q_{l-1}+(-1)^{l-1} p_{l-2} q_{l-2}}{2}\right)^{2} \\
& >2 \operatorname{deg} q_{l-1} \\
& >2 \operatorname{deg} q_{\frac{l}{2}-1}
\end{aligned}
$$

and by Lemma 2.2, we have

$$
\operatorname{deg} D>\operatorname{deg} u_{D}
$$

Suppose that $\operatorname{deg} w q_{l-1}=\operatorname{deg} p_{l-2} q_{l-2}$. Then we can write

$$
(-1)^{l-1} p_{l-2} q_{l-2}=\alpha q_{l-1}+\gamma
$$

for some $\alpha, \gamma \in k[x]$ such that $\operatorname{deg} \gamma<\operatorname{deg} q_{l-1}$ or $\gamma=0$ and we have

$$
w q_{l-1}+(-1)^{l-1} p_{l-2} q_{l-2}=(w+\alpha) q_{l-1}+\gamma .
$$

So if $w \neq-\alpha$, then by Lemma 2.1 we also have $\operatorname{deg} D>2 \operatorname{deg} q_{l-1}$ and by Lemma 2.2 we have

$$
\operatorname{deg} D>\operatorname{deg} u_{D}
$$

Thus we proved that $\operatorname{deg} D>\operatorname{deg} u_{D}$ for all $D \in S_{k}\left(l ; a_{1}, \ldots, a_{l-1}\right)$ with one possible exception, which is the case $w=-\alpha$ and in this case $D$ has the least degree in $S_{k}\left(l ; a_{1}, \ldots, a_{l-1}\right)$. 


\section{Proof of Theorem 1.3}

Let $q$ be an odd prime and $k=\mathbb{F}_{q}$. Let $D \in k[x]$ be a monic square-free polynomial of even degree and $K=k(x)(\sqrt{D})$ the real quadratic function field over $k$. Let $h\left(O_{K}\right)$ be the ideal class number of $O_{K}$ and $h_{K}$ the divisor class number of $K$. Then we have

$$
h_{K}=R_{K} h\left(O_{K}\right)
$$

where $R_{K}$ is the regulator of $K$. If $\epsilon_{K}=t_{D}+u_{D} \sqrt{D}$, then $R_{K}=\operatorname{deg} t_{D}$. We note that if $\operatorname{deg} D=2 N$, then the genus $g_{K}$ of $K$ is equal to $N-1$.

Let $l>1$ be a positive integer and $a_{1}, \ldots, a_{l-1}$ a palindromic sequence of non-constant polynomials in $k[x]$. Suppose that $D \in S_{k}\left(l: a_{1}, \ldots, a_{l-1}\right)$ and $\sqrt{D}=\left[a_{0}, \overline{a_{1}, a_{2}, \ldots, a_{l-1}, 2 a_{0}}\right]$ for some $a_{0} \in k[x]$. Let $\frac{p_{i}}{q_{i}}$ be the $i$-th approximant of the continued fraction $\left[a_{0}, \overline{a_{1}, a_{2}, \ldots, a_{l-1}, 2 a_{0}}\right]$. If $D$ does not have the least degree in $S_{k}\left(l: a_{1}, \ldots, a_{l-1}\right)$, then $\operatorname{deg} D>2 \operatorname{deg} q_{l-1}$. So we have

$$
\operatorname{deg} D=2 N>2 \operatorname{deg} q_{l-1}=2\left(\operatorname{deg} a_{1}+\cdots+\operatorname{deg} a_{l-1}\right)
$$

and

$$
R_{K} \leq \operatorname{deg} p_{l-1}=\operatorname{deg} a_{0}+\operatorname{deg} a_{1}+\cdots+\operatorname{deg} a_{n-1}=N+\operatorname{deg} q_{l-1}<2 N .
$$

So we have the following lower bound of the ideal class number of $K=$ $k(x)(\sqrt{D})$ from the work of Madan and Queen [6] and the work of Feng and $\mathrm{Hu}[2]$.

$$
\begin{aligned}
h\left(O_{K}\right) & \geq \frac{(q-1)\left(q^{2 g_{K}-1}+1-2 g_{K} q^{\frac{2 g_{K}-1}{2}}\right)}{R_{K}\left(2 g_{K}-1\right)\left(q^{g_{K}}-1\right)} \\
& >\frac{(q-1)\left(q^{2 N-3}+1-2(N-1) q^{\frac{2 N-3}{2}}\right)}{2 N(2 N-3)\left(q^{N-1}-1\right)} .
\end{aligned}
$$

This lower bound implies the following proposition.

Proposition 3.1. Let $q$ be an odd prime and $k=\mathbb{F}_{q}$. Let $D \in k[x]$ be a monic square-free polynomial of even degree and $K=k(x)(\sqrt{D})$ the real quadratic function field over $k$. If $K$ is not minimal type and the ideal class number of $K$ is equal to one, then

$$
\operatorname{deg} D= \begin{cases}4,6,8,10, & \text { if } q=3 \\ 4,6, & \text { if } q=5 \\ 4, & \text { if } q=7 .\end{cases}
$$

In particular, if $q \geq 11$, then all real quadratic function fields with ideal class number one is of minimal type.

Remark. If $\operatorname{deg} D=2, D$ is not contained in $S_{k}\left(l: a_{1}, \ldots, a_{l-1}\right)$ for $l>1$. In this case, $K=k(x)(\sqrt{D})$ always has ideal class number one. 
Proof of Theorem 1.3. Theorem 1.3 follows from Proposition 3.1 and the table of class numbers of quadratic function field in [2]. The complete list of 6 real quadratic function fields over $k$ with ideal class number one that are not of minimal type is following.

\begin{tabular}{cccc}
\hline$q, \operatorname{deg} D$ & $D$ & $R$ & $h\left(O_{K}\right)$ \\
\hline$q=3, \operatorname{deg} D=4$ & $x^{4}+2 x^{2}+2$ & 2 & 1 \\
\hline$q=3, \operatorname{deg} D=6$ & $x^{6}+x^{4}+x^{3}+x^{2}+2 x+2$ & 3 & 1 \\
& $x^{6}+x^{4}+2 x^{3}+x^{2}+x+2$ & 3 & 1 \\
& $x^{6}+x^{5}+2 x^{3}+2 x^{2}+2$ & 5 & 1 \\
& $x^{6}+2 x^{5}+x^{3}+2 x^{2}+2$ & 5 & 1 \\
\hline$q=5, \operatorname{deg} D=4$ & $x^{4}+2$ & 2 & 1 \\
\hline
\end{tabular}

\section{References}

[1] D. Byeon and S. Lee, A note on units of real quadratic fields, Bull. Korean Math. Soc. 49 (2012), no. 4, 767-774.

[2] K. Feng and W. Hu, On real quadratic function fields of Chowla type with ideal class number one, Proc. Amer. Math. Soc. 127 (1999), no. 5, 1301-1307.

[3] K. Feng and S. Sun, On class number of quadratic fields, Proceeding of First International Symposium on Algebraic Structures and Number Theory (1988, Hong Kong), Edited by S. P. Lam and K. P. Shum, World Scientific, (1990), 88-133.

[4] R. Hashimoto, Ankeny-Artin-Chowla conjecture and continued fraction, J. Number Theory 90 (2001), no. 1, 143-153.

[5] F. Kawamoto and K. Tomita, Continued fractions and certain real quadratic fields of minimal type, J. Math. Soc. Japan 60 (2008), no. 3, 865-903.

[6] M. Madan and C. Queen, Algebraic function fields of class number one, Acta Arith. 20 (1972), 423-432.

[7] J. Mc Laughlin, Multi-variable polynomial solutions to Pell's equation and fundamental units in real quadratic fields Pacific J. Math. 210 (2003), no. 2, 335-349.

[8] A. Stein, Introduction to continued fraction expansion in real quadratic function fields, Preprint.

DONGHO BYEON

Department of Mathematics

Seoul National University

SeOul 151-742, Korea

E-mail address: dhbyeon@snu.ac.kr

JiAe KeEM

Department of Mathematics

Seoul National University

SEOUl 151-742, KoreA

E-mail address: pure2942@snu.ac.kr

SAngyoon Lee

Department of Mathematics

Seoul National University

SeOul 151-742, Korea

E-mail address: lsyuis@hanmail.net 